\title{
Perceptual selectivity and the fate of unemphasized information in a stimulus complex
}

\author{
DONALD HOMA and BRUCE COURY \\ Arizona State University, Tempe, Arizona 85281
}

\begin{abstract}
The potential role of selectivity in visual perception was investigated for stimuli containing multiple components. Primary interest centered on the role of prior emphasis on the perceptual processing of one component, relative to the perceptual fate of the remaining components in the stimulus, and whether unemphasized components fared less well than those same components on an uninstructed trial. On each trial, a face, scrambled face, or single-component stimulus was briefly presented, followed by a patterned mask and a forced choice test of each of the components (eye, nose, mouth). On a cued trial, the subject was instructed to emphasize one component, although all three components were subsequently tested; on an uncued trial, no prior instructions were given. The results indicated that identification of an emphasized stimulus component was enhanced, but only at the detriment of the remaining components; that is, components were perceived more accurately on an uncued trial than unemphasized components on a cued trial. Although face and scrambled face stimuli were perceived unequally, the effectiveness of prior instructions was equivalent. Surprisingly, the overall identifiability of the components within a stimulus was independent of prior instructions, and suggested that a finite capacity is available for perceptual analysis.
\end{abstract}

The notion that a limitation exists in the processing of visual information is one that is rarely disputed. However, there is less agreement concerning the locus of this limitation; that is, at what point in the processing of visual information can an observer exercise selective control over some of the myriad of inputs constantly available, while ignoring the rest? The potential role of selectivity in perceptual processing was investigated in the present study by seeking clarification of the following questions: (1) When a subject is shown a stimulus complex whose component parts can vary, what is the effect of prior emphasis on one component relative to the remaining components in the complex? (2) Is performance worse on unemphasized components than on those same components when no prior information about the stimulus is provided? (3) Are stimuli which are more likely to be processed holistically or as a perceptual gestalt (Homa, Haver, \& Schwartz, 1976) less likely to be affected by prior emphasis on a single component than stimuli which are processed less efficiently? (4) Are the retention characteristics over short delays (less than $10 \mathrm{sec}$ ) different for emphasized and unemphasized information?

Theories of visual perception that conceive of selective attention as having a finite capacity that can be allocated to various locations within a visual display would predict that emphasizing instructions must result in a subsequent decrement in perceptual processing of

Requests for reprints should be sent to Donald Homa, Department of Psychology, Arizona State University, Tempe, Arizona 85281 . Portions of this experiment were presented to the Rocky Mountain Psychological Association, Phoenix, Arizona, May 1976. the unattended components (Neisser, 1967; Neisser \& Becklen, 1975; Rumelhart, 1970). Unlimited-capacity models (e.g., Gardner, 1973; Shiffrin \& Gardner, 1972; Shiffrin, McKay, \& Shaffer, 1976), which provide no attentional role in perception, would predict that prior emphasis on one component in a display would have little or no effect on the perceptibility of the unattended components. For example, in the independent-channels confusion model of Gardner (1973), it is argued that every feature in a stimulus has its own processing channel, where the processing efficiency for any one channel is independent of the processing efficiency for any other channel. According to the model, the decrement in performance typically found for increasing display sizes is attributed to the confounding influences of physical similarity. That is, physical similarity (confusion) among the elements in a display is likely to increase with increases in display size; it is this increased confusability, not any inherent limitations in perceptual processing, that is responsible for the observed decrement. In the present study, the number of components and the degree of confusability was held constant, so that subsequent differences in perceptual processing due to instructions would not be subject to this criticism.

The stimuli employed in the present study were identical to the schematic face, scrambled face, and single-component stimuli used earlier by Homa et al. (1976). The stimuli have a number of useful properties for investigating the perceptibility of components within a stimulus complex: (1) A single component, for example, eyes, can be emphasized prior to presentation, and accuracy for that component, as well as the remaining components (nose, mouth) can be measured 
and contrasted with uninstructed trials; (2) the number of components (eyes, nose, and mouth) can be held constant, while variations within each component can be manipulated separately; and, (3) stimuli may be employed which contain the same components but which differ in perceptual accuracy. In the study by Homa et al. (1976), it was found that perceptual accuracy was greater for schematic face than scrambled face stimuli. These differences were not due to factors such as differential guessing biases or forgetting, internal redundancy among the components, location uncertainty of the components, or familiarization of specific feature components. Since the components within a face were consistently perceived more accurately than the same components within a scrambled face, it was concluded that a schematic face functioned as a perceptual gestalt; that is, the arrangement of the components and not the redundancy of specific component variations was what mediated the face superiority. For the present study, an additional question of interest might be phrased as follows: If a schematic face functions as a perceptual gestalt, that is, if it is processed more as a unit, will prior emphasis of a component have less effect on the perceptibility of that component, as well as on the remaining components, than for the scrambled face stimuli? If a face is processed as a perceptual unit, then it might be expected that attentional mechanisms would be less able to "break up" a gestalt. For a scrambled face stimulus, whose components are processed independently, the role of prior emphasis on one component should be facilitatory for that component, but possibly at the detriment of the remaining components in the stimulus. Thus, it should also be expected that the remaining or unemphasized components would fare better for a face stimulus, relative to an uninstructed trial, than should those same components within a scrambled face stimulus.

The - present study might be contrasted with the role of prior information on perceptual selectivity studied in previous experiments. Egeth and Smith (1967) found that prior inspection of picture alternatives, one of which would be briefly shown, resulted in enhanced performance only when the alternatives were similar, for example, pictures of human dwellings such as a house, barn, church, and castle. They concluded that evidence for perceptual selectivity was most likely to be revealed only when the eventual discrimination was difficult; in other words, when stimulus alternatives are known beforehand, distinctive features can be extracted which discriminate among similar alternatives. However, Egeth and Smith's experiment was not designed to determine either the nature of the information used to discriminate the alternatives or the perceptual fate of less critical information in the stimulus. Again, a limited-capacity model would predict that whatever information was selectively used to eventually discriminate among the alternatives must be accompanied by a subsequent decrement on the remaining information. In a different paradigm, which involved the rapid sequencing of stimuli, Kubicek and Erdelyi (1974) found that priority instructions for items belonging to a common category (animals) facilitated recognition for stimuli belonging to that class, but not at the expense of the nonpriority stimuli. However, their study is mute with regard to the - effect of priority instructions on information within a stimulus.

In the present study, single-component, face, and scrambled face stimuli, composed of eye, nose, and mouth variations, were tachistoscopically presented under both cued and uncued conditions. On a cued trial, subjects were instructed to give emphasis to a particular component on the forthcoming trial (e.g., make certain that the type of eyes could be identified), even though the subject was aware that all three components would be tested (nose and mouth variations). On half the trials, the emphasized component was tested first, and, on the remaining cued trials, it was tested third, that is, after the two unemphasized components on the cued trial had been tested. On cued trials, it was possible, therefore, to directly compare the perceptual accuracy for emphasized and unemphasized components tested either first or third. All testing was accomplished by a variant of the forced choice procedure similar to that employed in word perception studies (e.g., Reicher, 1969), and required the subject to select a specific component variation from an array of five variations for that component. Comparison of cued-emphasized vs uncued components, tested either first or third, would indicate whether the emphasis instructions were facilitative; comparison of cuedunemphasized vs uncued components, tested first or third, would indicate the potential deleterious effect of emphasis instructions for one component on the remaining unemphasized components. A positive difference in the former case would be consistent with the view that subjects can use prior instructions to selectively analyze information in a multicomponent display; a negative difference in the latter case would argue that selective attention is a limited-capacity resource, that is, allocation of attention to an emphasized component resulted in enhanced accuracy for that component, but at the expense of the remaining information in the stimulus. Comparison of the magnitude differences for these two cases for face and scrambled face stimuli would allow a test of whether selective attention is more likely to be effective for a well organized (face) or a poorly organized (scrambled face) stimulus.

\section{METHOD}

\section{Subjects}

The subjects were 24 Arizona State University undergraduates from an introductory course in psychology. They participated in the experiment to partially fulfill the course requirement. Two subjects had to be replaced, one for failing 
to follow instructions properly and the other for having an excessively high error rate.

\section{Materials and Appara tus}

The three basic stimulus types, face, scrambled face, and single-component stimuli, were selected from the same stimulus pool employed earlier (see Figure 1, Homa et al., 1976). Briefly, a normal face was constructed by selecting one of five variations from each of the components (eyes, nose, and mouth), and assembling the variations in a head outline. A scrambled face was constructed by taking one of the face stimuli and realigning its component variations in the invariant order of nose at the top of the head outline, mouth in the middle, and eyes at the bottom. The single-component stimuli contained one of the variations of eyes, nose, or mouth, positioned in its appropriate location within the head outline.

A total of 120 experimental stimuli were generated: 45 faces, 45 scrambled faces, and 30 single components. For the faces and scrambled faces, each of the five variations for each of the three components was used equally often. The particular variations of eyes, nose, and mouth were selected semirandomly, with the restriction that all allowable pairwise combinations of components (eyes-nose, eyes-mouth, nose-mouth) were used at least once and no more than twice across the 45 stimuli. Thus, knowledge of any particular component variation (e.g., one of the variations of eyes) essentially provided for chance accuracy in terms of correctly guessing any other component variation (e.g., one of the variations of mouths).

In addition to the 120 stimuli, there were three basic test cards, labeled "Eyes," "Nose," and "Mouth." On the reverse side of each card were the five variations for that particular component. The component variations on the test cards were Xeroxed copies of the same variations which appeared on the stimulus cards. To facilitate scoring, each of the variations on a particular test card was associated with a number (1-5), and responses by the subject were always in the form of a number. An Iconix four-field tachistoscope (Model 6137-4) was used to present the stimuli.

\section{Procedure}

On each trial, one of three stimulus types (face, scrambled face, single component) was briefly presented, followed by a series of forced choice tests on each component in the stimulus. The subject was told that the components in a scrambled face would appear in the invariant order of nose, mouth, and eyes, from top to bottom; for single-component stimuli, one of the components would appear in its appropriate location within the outline of the head. On a cued trial, the subject was informed that one of the components was to be emphasized, even though, for face and scrambled face stimuli, each of the three components would be tested. On a cued trial, the subject was simply told to identify the emphasized component as well as possible, where an emphasized component could be treated as more valuable than the other components. It was stressed, however, that the subject should always fixate the centrally located dot (which preceded each stimulus), regardless of instructions. ${ }^{1}$

Prior to presentation of the stimuli, each subject was allowed to inspect each of the 15 component variations contained on the test cards. To insure that the subject understood the nature of the task, 30 practice stimuli, composed of the same component variations as the experimental stimuli, but otherwise different in terms of the particular combinations of component variations used, were presented at an initial exposure duration of $60 \mathrm{msec}$. Within each block of 15 practice trials, each of the cuing conditions and stimulus types occurred at least once. The practice trials also provided an opportunity to slightly adjust the exposure duration: If more than 10 of the 15 practice stimuli were answered incorrectly on the first of the three tests. the exposure duration was increased by $10 \mathrm{msec}$; if tive or less errors occurred, the exposure duration was decreased by $10 \mathrm{msec}$. A similar procedure was followed on the second block of practice stimuli, and allowed a potential exposure duration of 40-80 msec.

Following the practice trials, and if the subject had no questions, the presentation of the experimental stimuli was begun. On cued trials, the experimenter stated such and indicated which of the components was to be emphasized (e.g., "Face, Emphasize Eyes"). If the trial was uncued, the experimenter simply stated "Ready." All trials were initiated by the experimenter. The sequence of events on each trial was: the appearance of a small fixation point, centered in the field for $1 \mathrm{sec}$, followed by the presentation of the stimulus (for $60 \mathrm{msec}$, unless the adjustment indicated above was used), followed immediately by a $2-\mathrm{sec}$ visual noise mask. The masking field was composed of a random collection of bits and pieces of the 15 component variations which covered the entire stimulus configuration. Following offset of the mask, the subject was directed to turn over one of the three test cards before him, and to indicate which of the five variations for that particular component had been presented. For face and scrambled face stimuli, the procedure was repeated twice more, to complete testing on the remaining components in the stimulus. Subjects were always told to guess, even if they were totally uncertain of the correct response. During the session, the subject sat in a semidarkened room. Each session lasted about $1 \mathrm{~h}$.

\section{Design}

A within-subjects design was used, with the major variables being:(1) stimulus type (face, scrambled face, single component), (2) component tested (eyes, nose, mouth), (3) cuing condition (prior information about the stimulus type and component to be emphasized vs uncued trials in which no prior information was given), (4) order of test (components tested first, second, or third), and, on cued trials, (5) component value (cued-emphasized, cued-unemphasized). On cued trials, emphasized information was tested first or third (last) equally often, whereas uncued trials and unemphasized components on cued trials were tested at all three levels of test (first, second, and third). The 45 trials containing either face or scrambled face stimuli were composed of 15 cued trials in which the emphasized component was tested first, 15 cued trials in which the emphasized component was tested third, and 15 uncued trials. The 30 trials containing only single-component stimuli were equally divided into 15 cued and 15 uncued trials. Obviously, the order of test of single-component stimuli was always first.

For a block of eight subjects, the 120 experimental stimuli were randomized for presentation, with the order of presentation of the 120 stimuli rotated about every 15 stimuli for each subject. For the other two blocks of eight subjects, the stimuli were randomized again, and the starting order was rotated across the eight subjects. Across the 24 subjects, each of the $\mathbf{4 5}$ face and scrambled face stimuli occurred equally of ten under each of the cuing conditions and order of test.

\section{RESULTS}

The average exposure duration for the experimental stimuli was $64 \mathrm{msec}$, with an individual subject range of $50-80 \mathrm{msec}$. This resulted in a mean error rate of .504 , with about $70 \%$ of the subjects within an error range of .45-.60 (chance would be .80 ). No subject had an error rate of less than .25 , nor did any subject exceed an error rate of .64 .

For each subject, errors were tabulated for each combination of cuing condition (cued-emphasized, 


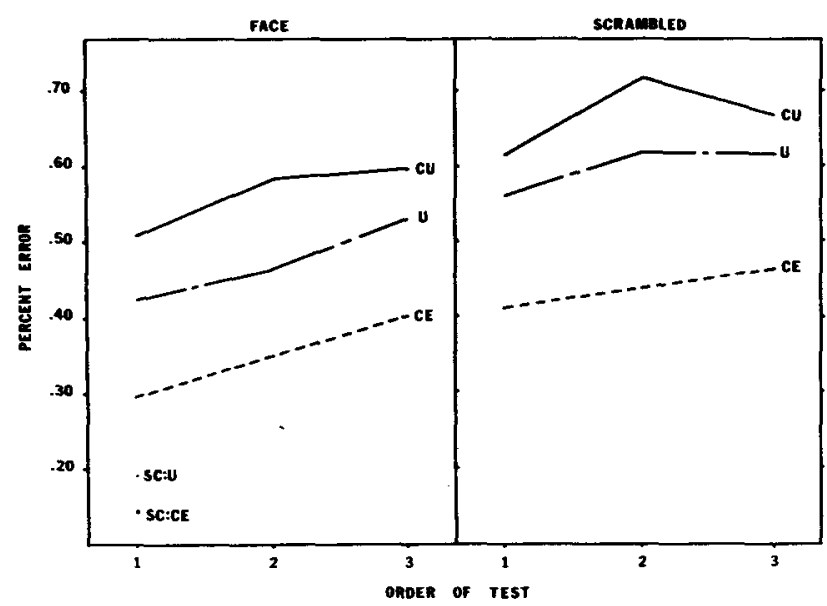

Figure 1. Mean error rates as a function of cuing condition and order of test for face, scrambled face, and single-component stimuli $\quad(C U=$ cued-unemphasized; $\quad C E=$ cued-emphasized; $\mathrm{U}=$ uncued; SC:CE = single-component stimulus, cuedemphasized).

cued-unemphasized, uncued), stimulus type (face, scrambled face, single component), component tested (eyes, nose, mouth), and order of test (first, second, third). Figure 1 shows the mean error rate as a function of cuing condition and order of test for face and scrambled face stimuli separately; performance on the single-component stimuli is included in the left panel of Figure 1 for the cued-emphasized and uncued trials.

Overall, the mean error rates for face, scrambled face, and single-component stimuli were $.484, .599$, and .167 , respectively. If only the performance on the first test, uncued trials, is considered, the corresponding error rates are $.422, .561$, and .192 . The differences across the three stimulus types are roughly similar to that obtained in an earlier study (Homa et al., 1976, Experiment 1). Thus, the random mixing of cuing instructions in a within-subjects design and the subject's awareness that all three components would be tested for face and scrambled face stimuli did not alter performance differences found in that earlier study. As indicated in Figure 1, the effects of cuing were similar for face and scrambled face stimuli, with cuedemphasized, cued-unemphasized, and uncued instructions resulting in overall error rates of $.347, .562$, and .471 , respectively, for face stimuli, and $.438, .679$, and .599 for scrambled face stimuli. Although performance tended to decrease across test order under all cuing conditions, the effectiveness of cuing was maintained for both face and scrambled face stimuli at each test order.

Since all levels of the major variables were not completely crossed (e.g., order of test with stimulus type), three separate analyses were computed on partially overlapping subsets of the data matrix where crossing did occur. In the first analysis, the effects of cuing conditions (cued-emphasized, cued-unemphasized, uncued) were contrasted for faces and scrambled faces under the first and third tests. The analysis revealed that all three major variables were highly significant: Components in faces were perceived more accurately than in scrambled faces $[\mathrm{F}(1,23)=43.38, \mathrm{MSe}=3.50]$, performance was worse on Test Trial 3 than on Trial $1 \quad[\mathrm{~F}(1,23)=18.46, \quad \mathrm{MSe}=5.37]$, and the three cuing conditions differed among themselves $[\mathrm{F}(2,46)=53.31, \mathrm{MSe}=4.42] \quad(\mathrm{p}<.001$ in each case $)$. A mild Stimulus Type by Test Order interaction was found $[\mathrm{F}(1,23)=3.55, \quad \mathrm{MSe}=2.35, .05<\mathrm{p}<.10]$ and reflected the slightly greater increase in errors for face stimuli from the first to the third test, as compared to scrambled faces. The mild interaction may be partially due to a ceiling effect on the already high error rates for scrambled faces on Test 1 . No other interactions were significant.

In a second analysis, all three levels of test order were compared for face and scrambled face stimuli under the uncued and cued-unemphasized trials only. The overall superiority of $8.6 \%$ on uncued relative to cued-unemphasized trials was highly significant $[F(1,23)=26.81, \mathrm{MSe}=3.59, \mathrm{p}<.001]$. As before, the effect of stimulus type $[F(1,23)=57.65$, MSe $=3.84]$ and order of test $[\mathrm{F}(2,46)=16.25, \mathrm{MSe}=2.38]$ were again highly significant (both $\mathrm{p}<.001$ ). None of the interactions were significant. Thus, unemphasized information on a cued trial was not perceived as well as when the trial was uncued; this outcome was found across all three test trials, and was not diminished by multiple tests.

In the final analysis, all three stimulus types (face, scrambled face, single component) were contrasted under the cued-emphasized and uncued conditions for the first test only. As expected, the three stimulus types differed among themselves $[\mathrm{F}(2,46)=129.86, \quad \mathrm{MSe}=2.15, \quad \mathrm{p}<.001], \quad$ with performance on single-component stimuli exceeding that for either face or scrambled faces $(.167, .358$, and .486 , respectively). The effect of cuing was highly significant $[F(1,23)=43.95, \quad \mathrm{MSe}=2.20, \quad \mathrm{p}<.001], \quad$ with performance under the cued-emphasized instructions resulting in an approximate $14 \%$ facilitation relative to an uncued trial. Although face stimuli were affected less by the prior emphasis instructions than were scrambled faces (cued-emphasized - uncued $=.124$ for faces and .161 for scrambled faces), a subsequent $t$ test failed to show the difference to be significant $(t<1)$. Individually, the emphasis instructions were more facilitative for scrambled faces than for faces for 12 subjects, 7 subjects showed the reverse, and 5 subjects showed no difference. A significant Stimulus Type by Cuing Condition interaction $[F(2,46)=3.89$, $\mathrm{MSe}=1.91, \mathrm{p}<.05$ ) resulted from the greater facilitation afforded face and scrambled faces due to emphasis instructions than for the single-component stimuli. 
Table 1

Mean Error Rate for Each Component as a Function of Cuing Condition, Stimulus Type, and Test Order

\begin{tabular}{|c|c|c|c|c|c|c|c|c|c|c|c|c|c|}
\hline $\begin{array}{c}\text { Test } \\
\text { Order }\end{array}$ & Cuing Condition & \multicolumn{4}{|c|}{ Face } & \multicolumn{4}{|c|}{ Scrambled } & \multicolumn{4}{|c|}{ Single Component } \\
\hline First & $\begin{array}{l}\text { Cued-Emphasized } \\
\text { Cued-Unemphasized } \\
\text { Uncued }\end{array}$ & $\begin{array}{l}.167 \\
.483 \\
.317\end{array}$ & $\begin{array}{l}.583 \\
.700 \\
.675\end{array}$ & $\begin{array}{l}.133 \\
.342 \\
.275\end{array}$ & $\begin{array}{l}.294 \\
.508 \\
.422\end{array}$ & $\begin{array}{l}.275 \\
.508 \\
.483\end{array}$ & $\begin{array}{l}.525 \\
.667 \\
.675\end{array}$ & $\begin{array}{l}.433 \\
.658 \\
.525\end{array}$ & $\begin{array}{l}.411 \\
.611 \\
.561\end{array}$ & $\begin{array}{l}.133 \\
.183\end{array}$ & $\begin{array}{l}.167 \\
.258\end{array}$ & $\begin{array}{r}.125 \\
.133\end{array}$ & $\begin{array}{l}.142 \\
.192\end{array}$ \\
\hline Second & $\begin{array}{l}\text { Cued-Emphasized } \\
\text { Cued-Unemphasized } \\
\text { Uncued }\end{array}$ & $\begin{array}{l}.508 \\
.350\end{array}$ & $\begin{array}{l}.729 \\
.667\end{array}$ & $\begin{array}{l}.479 \\
.367\end{array}$ & $\begin{array}{l}.572 \\
.461\end{array}$ & $\begin{array}{l}.654 \\
.533\end{array}$ & $\begin{array}{l}.804 \\
.767\end{array}$ & $\begin{array}{l}.696 \\
.558\end{array}$ & $\begin{array}{l}.718 \\
.619\end{array}$ & & & & \\
\hline
\end{tabular}

The previous analyses were collapsed across performance on the individual components (eyes, nose, mouth). Table 1 shows the mean error rate for these components as a function of cuing, stimulus type, and test order. First, the performance on each component was consistently determined by cuing condition, with cued-emphasized instructions always resulting in the greatest accuracy, cued-unemphasized always resulting in the worst performance, and uncued always intermediate. Thus, overall performance for each stimulus type under the various cuing conditions was consistently reflected in the performance of the individual components. Second, performance on each component was approximately the same for the singlecomponent stimuli; however, perceptibility of the nose component, when embedded in a face or scrambled face, was much worse, that is, perceptibility of the three components interacted with the stimulus type which contained them. Surprisingly, this outcome was largely unaffected by emphasis instructions, in that the uneven difficulty of the components in face and scrambled face stimuli was not diminished by emphasis instructions. In fact, the greatest benefactors of the emphasis instructions appeared to be those components which were more easily perceived under the uncued conditions. Third, performance on the eyes and mouth components for the face stimuli, when those components were emphasized, approximated the accuracy for those components when shown as a single-component stimulus. This was not the case, however, for the nose component.

Finally, it is possible to determine whether the perceptibility of the entire stimulus was enhanced, worsened, or unaffected by the cuing manipulations. If perception can be conceived as having a finite resource which can be allocated according to prior instructions, then a limited-capacity view of perception must predict that overall accuracy of the stimulus should be independent of prior instructions. By averaging performance on the emphasized component with the weighed average of the remaining components in the stimulus configuration (face or scrambled), an overall estimate of the perceptual accuracy under these instructions can be determined and compared to the uncued trials. For face stimuli, these values were .437 for a cued trial and .422 for an uncued trial (first test only), and .531 cued and .531 uncued (third test). For scrambled face stimuli, the overall accuracy on an emphasized trial was .544 vs .561 uncued (first test), and .601 cued vs .617 uncued (third test). Taken together, the results suggest that the overall accuracy on a cued trial (emphasized and unemphasized information) was about the same as when the trial was uncued, for both face and scrambled face stimuli, an outcome which might be predicted by a limited-capacity view of perceptual attention.

\section{DISCUSSION}

The major conclusions may be summarized as follows: (1) With instructions to emphasize a single component within a stimulus complex, identification of that component variation is enhanced; (2) the magnitude of the decrement of the unemphasized components is consistent with the view that a reservoir of finite capacity is available for perceptual analysis which can be allocated (or not) to components within a stimulus complex; and, (3) the effectiveness of instructions to emphasize a component is independent of the processing efficiency of a given stimulus. These conclusions receive support from results showing that instructions to emphasize a particular component reliably enhanced the identifiability of that component, but at the detriment of the remaining components within the stimulus. Compared with uncued trials, the size of the enhancement for emphasized information and the size of the corresponding decrement for unemphasized information was roughly the same for face and scrambled face stimuli, even though these stimulus types differed substantially in terms of perceptual accuracy. Perhaps the most surprising result was the apparent compensatory outcome of the emphasis instructions: Regardless of whether a trial was uncued or whether a component was emphasized, the overall identifiability of the components within the stimulus was the same. In effect, prior instructions 
were unable to improve the processing efficiency of the entire stimulus. However, the subject could determine the patterning of accuracy for components within the stimulus. Taken together, the results support the view that attentional mechanisms are not only operative in perceptual processing, but that this resource is allocatable. It should be noted that a major criticism applied to previous studies purporting to demonstrate selective attention, for example, the confounding of display size with degree of confusability (Gardner, 1973), is not relevant to the present study. Here, the effects of cuing were obtained for a display of constant size (faces and scrambled faces contained three components), and confusability among the components was balanced for both stimulus types and cuing conditions.

The results of the present study might be contrasted with other experiments which differ in interpretation as well as methodology. Since Estes (1972), Gardner (1973), and Shiffrin (Shiffrin \& Gardner, 1972; Shiffrin, Gardner, \& Allmeyer, 1973) have argued for a nonattentional view of perceptual processing, it might be asked how the interpretation of the present study can be reconciled with these conflicting views. One objection is that the results of the present study reflect influences other than perceptual ones (e.g., decisional or memorial effects subsequent to perceptual analysis). In effect, it would have to be argued that the effects of cuing did not alter perceptual processes but some later stage in processing. Although unrealized contaminating influences may exist which produced the cuing differences, it is clear that factors such as forgetting, guessing bias; differential component redundancy and confusability, and display size are incapable of explaining the effect of cuing. For example, the magnitude of the cuing manipulations was obtained on the initial test following stimulus exposure, and subsequent forgetting of component information was relatively constant for the three cuing conditions (e.g., decrements from the first to the third test averaged about $10 \%$, regardless of whether the trial was cued or not, or whether the component was emphasized or unemphasized). Thus, forgetting did occur across the multiple testing of components, but since cuing differences were obtained on the initial test and maintained equally across additional testing, it seems implausible that different memorial or decisional processes occurred prior to the first test and were maintained at a uniform rate thereafter.

An alternative view is that the results of the present study represent the legitimate influences of selective attention due to instructional set, and that results frequently taken as support for a nonattentional interpretation of perception are spurious. In this regard, two commonly employed paradigms which have produced results consonant with a nonattentional interpretation of perception, (1) a detection task involving simultaneous vs sequential presentation of stimulus elements and (2) detection tasks which manipulate display size, may be inappropriate for gauging the properties of perception or may produce misleading conclusions. For example, Shiffrin and his associates (e.g., Shiffrin \& Gardner, 1972; Shiffrin et al., 1973) have demonstrated that simultaneous presentation of four elements results in the detection of a target with an accuracy that is equivalent to the sequential presentation of the same elements. The assumption is made that attention, if operative in perception, should be able to be switched during sequential presentation, and, therefore, overall performance should be better than for simultaneous presentation. Since these modes of presentation have consistently failed to produce significant differences, it has been argued that attentional mechanisms must exist after perceptual processing has been completed. However, attentional mechanisms could exist in the simultaneous presentation, as evidenced in the present study, obviating the comparison between simultaneous and sequential presentation. It is necessary to assume that preattentive processes operate initially over the entire stimulus complex, providing global and contrastive input regarding the general characteristics of the display, for example, line shapes, orientation (Beck \& Ambler, 1973), etc. Following the initial processing, attentional mechanisms may then be focused upon "likely bets" (e.g., the target in the upper right quadrant) for a more complete analysis. The sequential presentation, by contrast, could not afford the same initial preattentive stage, since only one element is presented at a time. Furthermore, subsequent presentation of stimulus elements may be competitive or disruptive with the analysis of the current element. ${ }^{2}$ Essentially, it may be the case that both preattentive and attentional processes are operative during the simultaneous presentation, whereas the sequential presentation precludes the initial preattentive analysis and/or invokes extraneous interruptive processes. Although the present paradigm and the detection task employed by Shiffrin seem, on the surface, quite different, the inclusion of a manipulation used in the present task for their paradigm would provide a test of whether selective attention is operating within the simultaneous presentation. If the foils in the simultaneous task could be maintained at a low level of confusability with the target, but were otherwise different within a given display (rather than, say, all " $O$ "), cuing of the nontarget locations should determine whether all locations were processed equally well. The results of the present experiment, which involved cuing for both emphasized and unemphasized information, would predict that identification of nontargets would be inferior to that of target identification.

The nonattentional interpretation provided by Gardner (1973) could also be criticized on similar grounds. Evidence for parallel processing may reflect a preattentive perceptual stage (Neisser, 1967), but 
leave unanswered whether later perceptual processing exists and whether the later perceptual stages operate serially. By maintaining a constant and low level of confusability between target and foils, Gardner found that letter detection was unrelated to increases in display size. However, it may be necessary to manipulate stimulus complexity independently of target-nontarget confusability, since the resulting performance may be due to a preattentive (and parallel) analysis. ${ }^{3}$ Since others have found that prior cuing is facilitative for complex stimuli (Egeth \& Smith, 1967), but not for simple ones (Gummerman, 1970), it may be that selective attention need operate only when the stimulus elements become sufficiently complex. In effect, it seems indisputable that some parallel processing is likely in the analysis of stimulus inputs. This is not to say, however, that all perceptual processing need operate in parallel, especially if focal attention follows a parallel preattentive analysis (Neisser, 1967). The results of the present study argue strongly for a selective mechanism which operates during perception, where the allocation of fine-grained analysis for part of a stimulus can occur only with a concomitant decrement for the remaining portions of the stimulus complex.

Finally, some secondary results of the present study are worth noting. Since cuing for form class alone was ineffective in an earlier study (Homa et al., 1976, Experiment 1), it seems clear that perceptual set is unlikely to be demonstrated until some degree of stimulus specificity is provided. Also the deterioration of performance across the three component tests cannot be attributed to a simple decay interpretation, since an unfilled delay results in an enhancement, rather than a deterioration of performance (Homa et al., 1976, Experiment 3).

\section{REFERENCES}

Beck, J., \& AMBler, B. The effects of concentrated and distributed attention on peripheral acuity. Perception \& Psychophysics, 1973, 14, 225-230.

Ege Th, H., Jonides, J., \& Wall, S. Parallel processing of multielement displays. Cognitive Psychology, 1972, 3, 674-698.

Egeth, H., \& Smith, E. E. Perceptual selectivity in a visual recognition task. Joumal of Experimental Psychology, $1967,74,543-549$.

Estes, W. K. Interaction of signal and background variables in visual processing. Perception \& Psychophysics, 1972, 12, 278-286.

GARDNER, G. T. Evidence for independent parallel channels in tachistoscopic perception. Cognitive Psychology, 1973, 4, 130-155.

GUMMERMAN, K. Knowledge of alternatives and perception of tachistoscopic stimuli. Journal of Experimental Psychology, 1970, 83, 385-390.

Homa, D., Haver, B., \& Schwartz, T. Perceptibility of schematic face stimuli: Evidence for a perceptual gestalt. Memory \& Cognition, 1976, 4, 176-185.

KubiceK, L. F., \& ERdelyi, M. H. Effects of priority instructions on processing hypercapacity sequential inputs of pictures and words. Joumal of Experimental Psychology, 1974, 103, 729-731.

NeISSER, U. Cognitive psychology. New York: AppletonCentury-Crofts, 1967.

Neisser, U., \& Becklen, R. Selective looking: Attending to visually specified events. Cognitive Psychology, 1975, 7, 480-494.

REICHER, G. M. Perceptual recognition as a function of meaningfulness of stimulus materials. Journal of Experimental Psychology, 1969, 81, 275-280.

RUMELhART, D. E. A multicompnent theory of the perception of briefly exposed visual displays. Journal of Mathematical Psychology, 1970, 7, 191-218.

ShIFFrin, R. M., \& GaRdner, G. T. Visual processing capacity and attentional control. Journal of Experimental Psychology, 1972, 93, 72-82.

Shiffrin, R. M., Garoner, G. T., \& Allmeyer, D. H. On the degree of attention and capacity limitations in visual processing. Perception \& Psychophysics, 1973, 14, 231-236.

Shiffrin, R. M., McKay, D. P., \& Shaffer, W. 0. Attending to forty-nine spatial positions at once. Journal of Experimental Psychology: Human Perception and Performance, 1976, 2, 14-22.

\section{NOTES}

1. Caution was taken to insure that subjects followed the instruction to concentrate their gaze on the fixation point prior to stimulus presentation. Although direct observation, such as monitoring eye movements, was not possible, indirect evidence that this instruction was obeyed is supported by subjects' reports as well as by data analyses. For example, an attempt to fixate that general location containing the emphasized component should have produced two outcomes: (1) poorer performance on an unemphasized component two locations removed from the "fixated" emphasized component, compared to an unemphasized component only one location removed, and (2) no difference on the centrally located component in a stimulus when that component was emphasized vs performance on that centrally located component on an uncued trial. With regard to the former, error rates for the unemphasized components which were one and two locations removed from the emphasized component were, for the first test only, .488 and .510 , respectively, a nonsignificant difference. With regard to the latter, emphasis on the centrally located nose component for face stimuli and emphasis on the centrally located mouth component for scrambled face stimuli enhanced performance for these components by $10 \%$, relative to the same centrally located components on an uncued trial. Finally, the present authors place little credence on the accuracy of subjects' reports. Still, it might be added that most subjects reported that fixation of the center dot and subsequently attending to the emphasized component was a relatively simple matter.

2. For example, in two of the three experiments which provided individual subject data (Shiffrin \& Gardner, Experiments 1 and 3), only 2 of the 13 subjects performed better under the sequential presentation, whereas other subjects sometimes provided substantial differences favoring the simultaneous presentation. A similar outcome was obtained in their second experiment, which reported only group averages. Admittedly, the differences were always small, but the impression is that the sequential presentation may be more difficult than the simultaneous presentation for reasons unrelated to the issue of parallel-serial processing.

3. The results of a study by Egeth, Jonides, and Wall (1972) are more difficult to reconcile with a preattentive interpretation of the display-size effect, especially Experiments 3 and 4 . In their experiments, reaction time (RT) was the primary 
dependent measure, and subjects were asked to detect the presence or absence of a target. In Experiments 3 and 4, the target could be any digit and the nontargets were letters. In Experiment 3, error rates for present responses increased with display size, so the resulting $\mathrm{RT}$ function may have reflected a speed-accuracy tradeoff. In Experiment 4, however, error rates and RT were both uniform across display size. A nonattentional interpretation for this paradigm is complicated, however, by the significant RT functions for target-absent responses and by the results of Experiment 5. In Experiment 5 , a substantial increase in RT was found when different subjects received Display Sizes 1 and 6.

(Received for publication August 19, 1976; revision accepted November $28,1976$. ) 